\title{
UNIFORMIZATION IN A PLAYFUL UNIVERSE
}

\author{
BY YIANNIS N. MOSCHOVAKIS ${ }^{1}$
}

Communicated by Paul J. Cohen, February 26, 1971

It was shown in [1] and [3] that several questions about projective sets can be answered if one assumes the hypothesis of projective determinacy. We show here (in outline) that the same hypothesis settles the questions of uniformization and bases for all analytical classes.

Let $\omega=\{0,1,2, \cdots\}, R={ }^{\omega} \omega$ (the "reals"), $\mathscr{X}=X_{1} \times \cdots \times X_{k}$ with $X_{i}=\omega$ or $X_{i}=R$ be any product space. We study subsets of these product spaces, i.e. relations of integer and real arguments.

THEOREM 1. Let $n$ be odd, $n \geqq 1$, assume that every $\Delta_{n-1}^{1}$ game is determined. Then for each $\Pi_{n}^{1}$ relation $P \subseteq R \times \Upsilon$, there exists a $\Pi_{n}^{1}$ relation $P^{*} \subseteq P$ such that

$$
(\exists \alpha) P(\alpha, x) \Leftrightarrow(\exists ! \alpha) P^{*}(\alpha, x) .
$$

(For $n=1$ this is the classical Kondo-Addison Uniformization Theorem, see [8].)

There are many consequences of this result which are well known. The following computation of bases is the corollary which is foundationally most significant.

THEOREM 2. If every projective game is determined, then every nonempty analytical set has an analytical element.

More specifically: if $n$ is even, $n \geqq 2$, and every $\mathbf{\Delta}_{n-2}^{\mathbf{1}}$ game is determined, then every nonempty $\Sigma_{n}^{1}$ subset of $R$ contains a $\Delta_{n}^{1}$ real; if $n$ is odd, $n \geqq 1$, and every $\Delta_{n-1}^{1}$ game is determined, then there is a fixed real $\alpha_{0}$ such that the singleton $\left\{\alpha_{0}\right\}$ is $\Pi_{n}^{1}$ (so that $\alpha_{0}$ is $\Delta_{n+1}^{1}$ ) and every nonempty $\Sigma_{n}^{1}$ subset of $R$ contains a real recursive in $\alpha_{0}$.

(For $n=3$, this gives the Martin-Solovay Basis Theorem [5] with Mansfield's improvement [2]. The proofs in these two papers use only the fairly weak hypothesis that there exists a measurable cardinal, or even that for each $\alpha, \alpha^{\#}$ exists. Our proof depends on the determinacy of a particular $\Delta_{2}^{1}$ game and it can be verified that this game is determined if for every $\alpha, \alpha^{\#}$ exists.)

AMS 1970 subject classifications. Primary 04A15; Secondary 02F35.

Key words and phrases. Analytical relations, uniformization, bases.

1 The author is a Sloan Foundation Fellow. During the preparation of this paper he was partially supported by NSF Grant \#GP-22937. 
Our methods combine easily with methods developed by D. A. Martin [4] to yield the following additional result.

THEOREM 3. Let $n$ be odd, $n \geqq 1$, let $\boldsymbol{\delta}_{n}^{1}$ be the supremum of the lengths of the prewellorderings in $\boldsymbol{\Delta}_{n}^{1}$, assume that every $\Delta_{n-1}^{1}$ game is determined. If $B_{n}$ is the smallest Boolean algebra of sets containing the open sets and closed under $\left\langle\boldsymbol{\delta}_{n}^{1}\right.$ unions, then $\boldsymbol{\Delta}_{n}^{1} \subseteq B_{n}$ and each $\mathbf{\Sigma}_{n+1}^{1}$ set is the union of $\mathbf{\delta}_{n}^{1}$ sets in $B_{n}$.

(For $n=3$ this was shown by Martin in [4].)

In the proofs we use the axioms of Zermelo-Fraenkel set theory and the axiom $D C$ of dependent choices, but not the full axiom of choice. Thus the results hold in the theory $Z F+D C+$ each game is determined. For this latter theory, Theorem 3 combines with results of Martin in [4] and ours [6] to give the elegant characterizations (odd $n$ ),

$$
\begin{gathered}
\boldsymbol{\Delta}_{n}^{1}=B_{n}, \\
P \in \Sigma_{n+1}^{1} \Leftrightarrow P=\bigcup_{\xi<\delta n}^{1} P_{\xi}, \quad \text { with each } P_{\xi} \in B_{n}{ }^{2}
\end{gathered}
$$

Full details will appear in [7].

1. Terminology. Precise definitions of the classes $\Sigma_{n}^{1}, \Pi_{n}^{1}$, etc., determinacy, the axiom $D C$ of dependent choices and recursive functions $f: x \rightarrow \mathcal{Y}$ with domain and range any product space can be found in [6].

If $P \subseteq \mathscr{X}$ is a pointset we also think of it as a relation and write interchangeably,

$$
x \in P \Leftrightarrow P(x) .
$$

It will be convenient to use "algebraic" notation for the logical opera-

${ }^{2}$ The exact computation of the ordinals $\delta_{n}^{1}(n \geqq 1)$ poses a very interesting problem. The following facts are known:

(1) In $Z F+D C, \boldsymbol{\delta}_{1}^{1}=\boldsymbol{N}_{1}$ (classical result).

(2) In $Z F+D C+F u l l$ Determinacy, $\aleph_{1}$ and $\aleph_{2}$ are measurable, hence regular (R. M. Solovay).

(3) In $Z F+D C+F u l l$ Determinacy, all $\delta_{n}^{1}$ are cardinals, $\delta_{n}^{1} \geqq \aleph_{n}$ and for odd $n, \delta_{n}^{1}$ is regular, [6].

(4) In $Z F+D C, \boldsymbol{\delta}_{2}^{1} \leqq \boldsymbol{N}_{2}$, hence in $Z F+D C+F u l l$ Determinacy, $\boldsymbol{\delta}_{\mathbf{2}}^{1}=\boldsymbol{N}_{2}$ (D. A. Martin, unpublished). [4]!

(5) In $Z F+D C+F u l l$ Determinacy, $\mathbf{\delta}_{\mathbf{3}}^{\mathbf{1}}=\mathbf{\aleph}_{\omega+1}=$ the first regular cardinal above $\boldsymbol{\aleph}_{\mathbf{2}}$,

(6) In $Z F+$ Projective Determinacy + Full Choice, $\delta_{3}^{1} \leqq \aleph_{3}$, [4].

(7) In $Z F+D C+F u l l$ Determinacy, for each odd $n, \delta_{n}^{1}=\left(\lambda_{n}\right)+$ for some cardinal $\lambda_{n}$ of cofinality $\omega$ (A. S. Kechris, unpublished, using the methods of the present note). 
tions on these pointsets, e.g.

$$
\begin{aligned}
& x \in \exists^{\omega} P \Leftrightarrow(\exists n) P(n, x) \quad(P \subseteq \omega \times x) \text {, } \\
& x \in \forall^{\omega} P \Leftrightarrow(\forall n) P(n, x) \quad(P \subseteq \omega \times X) \text {, } \\
& x \in \exists^{R} P \Leftrightarrow(\exists \alpha) P(\alpha, x) \quad(P \subseteq R \times x) \text {, } \\
& x \in \forall^{R} P \Leftrightarrow(\forall \alpha) P(\alpha, x) \quad(P \subseteq R \times x) .
\end{aligned}
$$

Similarly, if $\Gamma$ is a class of pointsets, $\exists^{R} \Gamma=\left\{\exists^{R} P: P \in \Gamma\right\}, \forall^{R} \exists^{R} \Gamma$ $=\left\{\forall^{R} \exists^{R} P: P \in \Gamma\right\}$, etc.

The dual class $\widetilde{\Gamma}$ is defined by $\breve{\Gamma}=\{x-P: P \in \Gamma\}$.

A class of pointsets $\Gamma$ is adequate if it contains all recursive pointsets and is closed under conjunction, disjunction, bounded number quantification and substitution of recursive functions. All classes $\Sigma_{n}^{1}, \Pi_{n}^{1}, \Delta_{n}^{1}$ are adequate, even with $n=0$ ( $\Sigma_{0}^{1}=$ all recursively enumerable sets).

For each $\Gamma$, let $\boldsymbol{\Gamma}$ be the class of all $P \subseteq x$ such that for some $Q \subseteq R \times X, Q \in \Gamma$ and some $\alpha_{0} \in R$,

Finally,

$$
P(x) \Leftrightarrow Q\left(\alpha_{0}, x\right) .
$$

$$
\boldsymbol{\Delta}=\{P \subseteq x: P \in \boldsymbol{\Gamma} \text { and } x-P \in \boldsymbol{\Gamma}\} .
$$

2. Norms and scales. The idea of the proof is to formulate a strong prewellordering property, like that of [1], which on the one hand can be shown to propagate from each $\Pi_{n}^{1}$ to $\Sigma_{n+1}^{1}$ and from each $\Sigma_{n+1}^{1}$ to $\Pi_{n+2}^{1}$, and on the other hand implies uniformization when it holds on a II-class.

A norm on a set $P$ is a function $\varphi: P \rightarrow$ ordinals; we call $\varphi$ a $\Gamma$-norm if there are relations $\leqq_{\Gamma}, \leqq \breve{\Gamma}$ in $\Gamma$ and $\breve{\Gamma}$ respectiveley, such that

$$
P(y) \Rightarrow(\forall x)[x \leqq \Gamma y \Leftrightarrow x \leqq \breve{\Gamma} y \Leftrightarrow[P(x) \& \varphi(x) \leqq \varphi(y)]] .
$$

$\Gamma$ has the prewellordering property in the sense of [1] or [3], if every $P \in \Gamma$ admits a $\Gamma$-norm.

A scale on a set $P$ is a sequence $\varphi_{0}, \varphi_{1}, \varphi_{2}, \cdots$ of norms on $P$ such that the following limit condition holds:

(*) If $x_{0}, x_{1}, x_{2}, \cdots \in P$, if $\operatorname{limit}_{i \rightarrow \infty} x_{i}=x$, if, for each $n$ and all

$$
\text { large } i, \varphi_{n}\left(x_{i}\right)=\lambda_{n} \text {, then } P(x) \text { and, for each } n, \varphi_{n}(x) \leqq \lambda_{n}{ }^{3}
$$

We call $\varphi_{0}, \varphi_{1}, \varphi_{2}, \cdots$ a $\Gamma$-scale if there are relations $S_{\Gamma}(n, x, y)$, $S_{\widetilde{\Gamma}}(n, x, y)$ in $\Gamma$ and $\breve{\Gamma}$ respectively, such that for each $n$,

' I wish to thank my student A. S. Kechris for simplifying my original definition of a scale and thereby shortening considerably the computation in the proof of $\mathrm{C}$ below. 


$$
P(y) \Rightarrow(\forall x)\left[S_{\Gamma}(n, x, y) \Leftrightarrow S_{\breve{\Gamma}}(n, x, y) \Leftrightarrow\left[P(x) \& \varphi_{n}(x) \leqq \varphi_{n}(y)\right]\right] .
$$

$\Gamma$ has property $s$ if each $P \in \Gamma$ admits a $\Gamma$-scale.

3. Basic results. Theorem 1 follows fairly easily from the following four basic results.

THEOREM A. The class $\Sigma_{0}^{1}$ of all recursively enumerable sets has property $s$.

Theorem B. If $\Gamma$ is adequate, $P \in \Gamma$ and $P$ admits a $\Gamma$-scale, then $\exists^{R} P$ admits $a \exists^{R} \forall^{R} \Gamma$-scale.

THeOREM C. If $\Gamma$ is adequate, if each $\Delta$ game is determined and $D C$ holds, if $P \in \Gamma$ admits a $\Gamma$-scale, then $\forall^{R} P$ admits a $\forall^{R} \exists^{R} \Gamma$-scale.

THEOREM D. If $\Gamma$ is adequate, $\exists^{\omega} \Gamma \subseteq \Gamma, \forall{ }^{\omega} \Gamma \subseteq \Gamma, \forall^{R} \Gamma \subseteq \Gamma$ and $\Gamma$ has property $s$, then for each $P \subseteq R \times x, P \in \Gamma$, there is some $P^{*} \subseteq P$ such that $P^{*} \in \Gamma$ and

$$
(\exists \alpha) P(\alpha, x) \Leftrightarrow(\exists ! \alpha) P(\alpha, x) .
$$

4. Proofs. Proof of $A$ is trivial and that of $D$ is a minor modification of a standard proof of the Kondo-Addison Theorem, e.g. that in [8]. Proofs of $\mathrm{B}$ and $\mathrm{C}$ are elaborations of the corresponding cases in the proof of the Prewellordering Theorem in [1]. We omit all details of $B$, which is the easier of the two.

To prove C, suppose

$$
P(x) \Leftrightarrow(\forall \alpha) Q(\alpha, x),
$$

with $Q \in \Gamma$, let $\psi_{0}, \psi_{1}, \psi_{2}, \cdots$ be a $\Gamma$-scale on $Q$. Let $u_{0}, u_{1}, u_{2}, \ldots$ be a recursive enumeration of all finite sequences of $\omega$ such that $u_{0}$ is the empty sequence and if $u_{i}$ is an initial segment of $u_{j}$, then $i<j$. For each $i$ and each $x, y$, consider the game $G_{i}(x, y)$ defined as follows: if player I plays $\gamma$ and player II plays $\delta$, put

$$
\alpha=u_{i} \cap \gamma, \quad \beta=u_{i} \cap \delta
$$

and call II a winner if one of the following conditions hold:

(0) $\neg Q(\beta, y)$,

(1) $Q(\beta, y) \& Q(\alpha, x) \& \psi_{0}(\alpha, x)<\psi_{0}(\beta, y)$,

(2) $Q(\beta, y) \& Q(\alpha, x) \& \psi_{0}(\alpha, x)=\psi_{0}(\beta, y) \& \psi_{1}(\alpha, x)<\psi_{1}(\beta, y)$,

$$
\begin{aligned}
Q(\beta, y) \& Q(\alpha, \dddot{x}) \& \psi_{0}(\alpha, x) & =\psi_{0}(\beta, y) \& \cdots \& \psi_{i-1}(\alpha, x) \\
& =\psi_{i-1}(\beta, y) \& \psi_{i}(\alpha, x) \leqq \psi_{i}(\beta, y) .
\end{aligned}
$$

For each $i$, put 


$$
\begin{gathered}
P_{i}(x) \Leftrightarrow\left(\forall \alpha \supseteq u_{i}\right) Q(\alpha, x), \\
x \leqq_{i} y \Leftrightarrow x, y \in P_{i} \& \text { II wins } G_{i}(x, y) .
\end{gathered}
$$

Notice that $P_{0}=P$. Now the methods of [1] easily show that if every $\Delta$ game is determined, then each $\leqq_{i}$ is a prewellordering on $P_{i}$ and hence defines a norm $\varphi_{i}: P_{i} \rightarrow$ ordinals. Moreover, there are relations $S_{1}(n, x, y), S_{2}(n, x, y)$ in $\forall^{R} \exists^{R} \Gamma$ and $\exists^{R} \forall^{R} \breve{\Gamma}$ respectively, such that

$$
P_{n}(y) \Rightarrow(\forall x)\left[S_{1}(n, x, y) \Leftrightarrow S_{2}(n, x, y) \Leftrightarrow\left[P_{n}(x) \& \varphi_{n}(x) \leqq \varphi_{n}(y)\right]\right] .
$$

The sequence $\varphi_{0}, \varphi_{1}, \varphi_{2}, \cdots$ consists of norms on different sets, but it is not hard to verify that if we can show the limit property (*) for it, then we can define a scale $\varphi_{0}^{\prime}, \varphi_{1}^{\prime}, \varphi_{2}^{\prime}, \ldots$ on $P$ itself.

Let $x_{0}, x_{1}, x_{2}, \cdots \in P$, assume that $\operatorname{limit}_{i \rightarrow \infty} x_{i}=x$ and for each $n$ and all large $i, \varphi_{n}\left(x_{i}\right)=\lambda_{n}$; we must show that $P(x)$ and for all $n$, $\varphi_{n}(x) \leqq \lambda_{n}$. Without loss of generality we may assume that $\varphi_{n}\left(x_{i}\right)=\lambda_{n}$, all $i \geqq n$; thus it is enough to show that, for each $i$, II has a winning strategy in $G_{i}\left(x, x_{i}\right)$, since for $i=0$ this proves $P(x)$ and for all $i$ it shows $x \leqq_{i} x_{i}$, i.e. $\varphi_{i}(x) \leqq \varphi_{i}\left(x_{i}\right)=\lambda_{i}$.

Suppose $u_{i}=\left(a_{0}, \cdots, a_{l}\right)$ and let us picture the game $G_{i}\left(x, x_{i}\right)$ as follows:

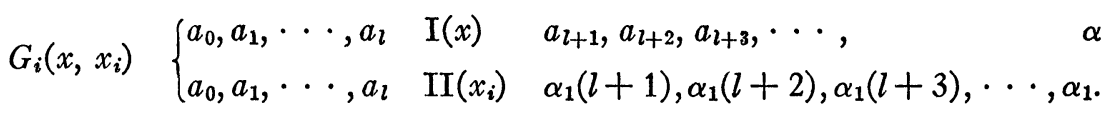

Here I's first move is labeled $a_{l+1}$, his second $a_{l+2}$, etc. Let $j_{1}, j_{2}, \ldots$ be chosen so that

$$
u_{j_{n+1}}=\left(a_{0}, a_{1}, \cdots, a_{l}, a_{l+1}, \cdots, a_{l+n}\right) ;
$$

notice that $i=j_{1}<j_{2}<j_{3}<\cdots$ and that $j_{n+1}$ is known as soon as $a_{l+n}$ has been played. For each $n$ then, II simulates on the side the game $G_{j_{n}}\left(x_{j_{n+1}}, x_{j_{n}}\right)$ in which the second player has a winning strategy. In all these simulated games, the second player follows some winning strategy. The first player starts with $a_{l+n}$ and then continues by copy-

\begin{tabular}{|c|c|c|}
\hline$G_{j_{1}}\left(x_{j_{2}}, x_{j_{1}}\right)\left\{\begin{array}{l}a_{0}, \cdots, a_{l} \\
a_{0}, \cdots, a_{l}\end{array}\right.$ & $\begin{array}{l}\mathrm{I}\left(x_{j_{2}}\right) \\
\mathrm{II}\left(x_{j_{1}}=x_{i}\right)\end{array}$ & $\begin{array}{l}a_{l+1}, \alpha_{2}(l+2), \alpha_{2}(l+3), \cdots, \\
\alpha_{1}(l+1), \alpha_{1}(l+2), \alpha_{1}(l+3), \cdots, \alpha_{l}\end{array}$ \\
\hline$G_{j_{2}}\left(x_{j_{2}}, x_{j_{2}}\right)\left\{\begin{array}{l}a_{0}, \cdots, a_{l}, a_{l+1} \\
a_{0}, \cdots, a_{l}, a_{l+1}\end{array}\right.$ & $\begin{array}{l}\mathrm{I}\left(x_{j_{3}}\right) \\
\mathrm{II}\left(x_{j_{2}}\right)\end{array}$ & $\begin{array}{l}a_{l+2}, \alpha_{3}(l+3), \cdots, \\
\alpha_{2}(l+2), \alpha_{2}(l+3), \cdots,\end{array}$ \\
\hline$\left(x_{j_{4}}, x_{j_{8}}\right)\left\{\begin{array}{l}a_{0}, \cdots, a_{l}, a_{l+1}, a_{l+2} \\
a_{0}, \cdots, a_{l}, a_{l+1}, a_{l+2}\end{array}\right.$ & $\begin{array}{l}\mathrm{I}\left(x_{j_{4}}\right) \\
\mathrm{II}\left(x_{j_{8}}\right)\end{array}$ & $\begin{array}{l}a_{l+3}, \cdots \\
\alpha_{3}(l+3), \cdots,\end{array}$ \\
\hline
\end{tabular}
ing the second player's moves in $G_{j_{n+1}}\left(x_{j_{n+2}}, x_{j_{n+1}}\right)$ as in the diagram below. Finally II copies the second player's move in $G_{j_{1}}\left(x_{j_{2}}, x_{j_{1}}\right)$ for the original game $G_{i}\left(x, x_{i}\right)$. 
At the end the second players have won all the simulated games and reals $\alpha, \alpha_{1}, \alpha_{2}, \alpha_{3}, \cdots$ have been defined. Clearly limit $i_{i \rightarrow \infty} \alpha_{i}=\alpha$, so that $\operatorname{limit}_{i \rightarrow \infty}\left(\alpha_{i}, x_{i}\right)=(\alpha, x)$. It is now easy to verify that all norms $\psi_{n}\left(\alpha_{i}, x_{i}\right)$ are constant for all large $i$, so that $Q(\alpha, x)$, and furthermore that II wins $G_{i}\left(x, x_{i}\right)$, thus completing the proof.

(ADDED IN PROOF, June 27, 1971.) K. Kunen and D. A. Martin have now shown, independently, in $Z F+D C+$ Projective Determinacy, that for each odd $n, \boldsymbol{\delta}_{n+1} \leqq\left(\delta_{n}\right)^{+}$; their proofs use the methods of this note. By entirely different methods D. A. Martin also showed in $Z F+D C+$ Full Determinacy, that for each odd $n, \boldsymbol{\delta}_{n}^{1}$ is measurable, and $\mathrm{K}$. Kunen showed that under the same hypotheses for all $n \geqq 1, \delta_{n}^{1}$ is measurable.

\section{BIBLIOGRAPHY}

1. J. W. Addison and Yiannis N. Moschovakis, Some consequences of the axiom of definable determinateness, Proc. Nat. Acad. Sci. U.S.A. 59 (1968), 708-712. MR 36 \#4979. 379.

2. Richard Mansfield, A Souslin operation for $\Pi_{2}^{1}$, Israel J. Math. 9 (1971), 367-

3. D. A. Martin, The axiom of determinateness and reduction principles in the analytical hierarchy, Bull. Amer. Math. Soc. 74 (1968), 687-689. MR 37 \#2607.

4. - Pleasant and unpleasant consequences of determinateness, unpublished manuscript, circulated in March 1970.

5. D. A. Martin and R. M. Solovay, $A$ basis theorem for $\Sigma_{\mathbf{3}}^{1}$ sets of reals, Ann. of Math. (2) 89 (1969), 138-159. MR 41 \#53.

6. Yiannis N. Moschovakis, Determinacy and prewellorderings of the continuum, Math. Logic and Foundations of Set Theory, North-Holland, Amsterdam and London, 1970, pp. 24-62.

7. - Descriptive set theory, a foundational approach, Studies in Logic, NorthHolland, Amsterdam (to appear).

8. Joseph R. Shoenfield, Mathematical logic, Addison-Wesley, Reading, Mass., 1967. MR 37 \#1224.

University of California, Los Angeles, California 90024 